\title{
Klassische Homöopathie: Das richtige Schmerzmittel finden
}

\begin{abstract}
Apis nimmt man bei Bienenstichen. Nux vomica bei Bauchschmerzen mit Durchfall und Erbrechen. Bei Schmerzen mit Fieber hilft Belladonna. Manche homöopathische Mittel haben sich bei der Behandlung bestimmter Schmerzzustände bewährt und werden häufig verordnet. Die Apothekerin und Heilpraktikerin Dr. Eva Lang zeigt anhand von Fallbeispielen aus ihrer Praxis, wie man in der Klassischen Homöopathie das jeweils wirksamste Schmerzmittel sicher bestimmt und einsetzt.
\end{abstract}

Wer kennt sie nicht: Schmerzen. Von der Schädeldecke bis zu den Zehenspitzen überall im Körper können sie auftreten. Brennen, stechen, hämmern, pochen, klopfen, krampfen - so verschieden können sie sich anfühlen. Die Ursachen: Prellungen, Verletzungen, Entzündungen, Bisse, Infektionen, Überlastung, Stress, Kummer etc. Die Liste scheint endlos. Und dann reagiert auch jeder Patient psychisch anders auf seine Schmerzen. Doch wie findet man für jeden Fall das richtige Schmerzmittel?

\section{Das „vollständige Symptom“}

Die Anamnese und erfolgreiche Behandlung von Schmerzen ist ein komplexes Thema. Es gibt keine Pauschallösungen. Von Fall zu Fall müssen wir individuell vorgehen, um unseren Patienten so wirkungsvoll wie möglich zu helfen. Doch ist nicht genau das die Stärke der Homöopathie?

In der Homöopathie verschreiben wir keine Arzneien nach Diagnosen, sondern widmen uns intensiv der Symptomatik. Dabei ist genau zu ermitteln:

- wo der Schmerz lokalisiert ist und wie er sich anfühlt

- seit wann er besteht und wodurch (Causa) er ausgelöst wurde (falls bekannt)

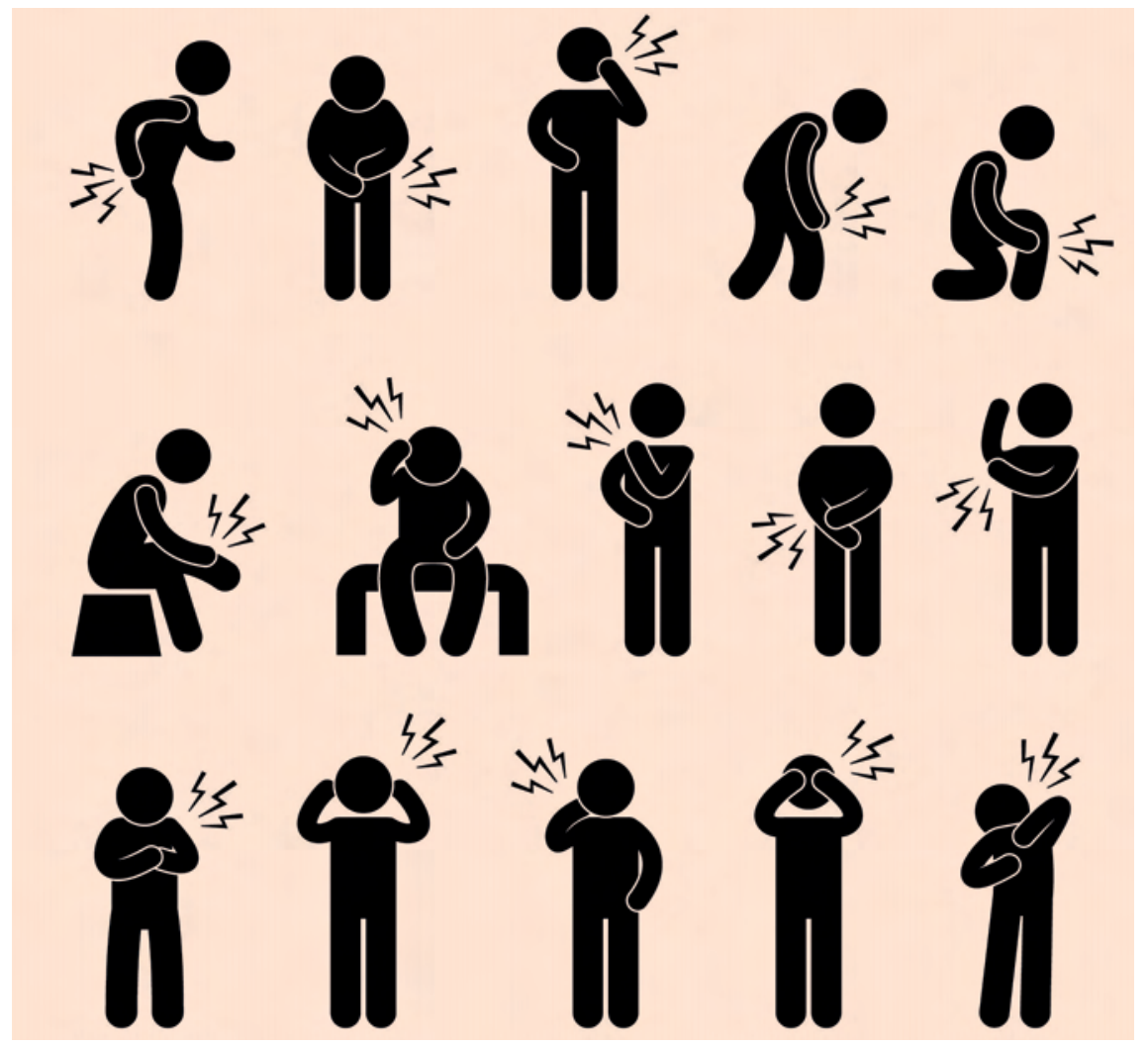

Abb. 1 Schmerz ist nicht gleich Schmerz: Er kann verschiedenste Ursachen haben, überall im Körper auftreten und fühlt sich jedes Mal anders an. Foto: @ Fotolia/leremy

- welche Situationen oder Handlungen den Schmerz verbessern oder verschlechtern

- ob andere Symptome ihn begleiten

- wie sich das psychische Befinden des Patienten durch den Schmerz verändert

Hahnemann nennt dies das Herausarbeiten des „vollständigen Symptoms“. Dabei spielt es keine Rolle, ob die Schmerzen akut oder chronisch sind. Es ist jedoch immer von Bedeutung, wodurch sie entstanden sind oder was ihnen vorausgegangen ist. Dabei kann die Ursache mechanisch (Stoß, Sturz), infektiös, aber auch psychisch (Stress, Kummer, Ärger) sein.
Auch Klima (z. B. Hitze, Kälte) oder bestimmte Nahrungs- und Genussmittel können Schmerzen verursachen.

\section{Homöopathie wirkt auf den ganzen Menschen}

Anhand kurz ausgeführter Fälle stelle ich im Folgenden einige häufig verordnete Mittel bei Abdominal-, Kopf-, Gelenk- und Rückenschmerzen vor. Dabei arbeite ich bei den einzelnen Fällen die Symptome heraus, die zum Auffinden der jeweiligen Arzneimittel bei akuten Erkrankungen wichtig sind. Beim Lesen wird Ihnen wahrscheinlich auffallen, dass sich die Symptome der Mittel wiederholen. Unabhängig davon, um welche Beschwerden es sich handelt. Das 
ist typisch für homöopathische Mittel: Ein Mittel zeichnet sich nicht dadurch aus, dass es auf ein bestimmtes Organsystem wirkt. Es wirkt auf den ganzen Menschen. Egal an welcher Stelle und an welchem Organ dieser erkrankt ist. So kann der Nachbar zur Rechten Nux vomica wegen seiner Herzbeschwerden bekommen und der zur Linken wegen seiner Bauchschmerzen. Die Homöopathie basiert auf der Überzeugung, dass Krankheiten ihren Ursprung nicht nur im Körper haben, sondern aus einer gestörten Lebenskraft heraus resultieren. Deshalb ist der ganze Mensch betroffen: mit Körper, Geist und Gemüt.

Dabei gilt es zu unterscheiden, ob der Mensch akut oder chronisch krank ist. Die Beschreibung der Arzneimittel für die chronische Behandlung würde jedoch den Rahmen sprengen. Daher werde ich mich hier auf die Behandlung von akuten Schmerzzuständen beschränken.

\section{Abdominalschmerzen - häufig Beschwerden der Eingeweide}

Chamomilla: Krampfhafte Schmerzen im Unterbauch an den ersten beiden Tagen ihrer Menses. Besserung durch Wärme, Verschlechterung nachts, aber auch morgens. Sie ist ziemlich wütend, unruhig und schreit bei der kleinsten Kleinigkeit los oder wirft Gegenstände durch die Gegend. Belladonna: Immer wieder krampfhafte Schmerzen im ganzen Bauch ohne ersichtlichen Grund. Sie kommen plötzlich und sind genauso plötzlich wieder weg. Dabei friert sie, hat kalte Hände und Füße, aber der Kopf ist heiß und rot. Wenn ihr kalt geworden ist, kommen die Schmerzen häufiger. Bauchlage oder bewegungslos liegen bessert, jede Bewegung und Erschütterung verschlimmert.

Colocynthis: Die Schmerzen sind krampfhaft, sie muss sich zusammenkrümmen und die Beine an den Bauch ziehen. Die Wärmflasche fest auf den Bauch drücken tut gut. Sie ist gereizt und schlägt bei
Schmerzen um sich. Ist in der Schule gemobbt worden.

Lycopodium: Die Schmerzen sind um den Bauchnabel herum, aber auch im ganzen Bauch. Wenn sie Blumenkohl oder Zwiebeln essen muss, bekommt sie Bauchschmerzen. Auch wenn sie eine Arbeit schreiben muss, steht sie morgens mit Bauchschmerzen auf. Meistens hat sie einen aufgetriebenen Leib und muss viel Winde lassen. Berührungen auf dem Bauch verschlechtern den Schmerz, warmer Tee ist angenehm. Bei Schmerzen duldet sie keinen Menschen in unmittelbarer Nähe. Sie kann dann ziemlich eklig zu ihrer Mutter sein.

Arsenicum album: Seit 2 Tagen hat sie Bauchschmerzen; gestern mit Erbrechen und Durchfall. Sie weiß nicht, wodurch die Beschwerden begonnen haben, aber nachts um 1:00 Uhr ist sie mit Erbrechen wach geworden. Sie ist erschöpft, friert und ist sehr blass. Der Durchfall stinkt wie Jauche und ist ganz dünn. Der After ist inzwischen rot und brennt. Sie mag nichts essen, aber kleine Schlückchen Wasser kann sie trinken. Sie ist sehr ängstlich und denkt immer, dass sie etwas ganz Schlimmes hat.

Kopfschmerzen - häufig nervale aber auch vaskuläre Beschwerden

Natrium muriaticum: Seit ca. 4 Wochen anhaltend hämmernde Kopfschmerzen, besonders in der Stirn. Er fühlt sich benommen, der Kopf ist schwer. Er kann nicht mehr denken. Um die Mittagszeit und in der Sonne wird es ganz schlimm, frische kühle Luft tut gut. Vor gut 5 Wochen musste er seinen kranken Hund einschläfern lassen. Lycopodium: Seit ein paar Tagen stechende Kopfschmerzen an der rechten Schläfe. Nachmittags sind sie schlimmer. Er hat zwar schon immer Last mit Blähungen gehabt, aber jetzt sind sie unerträglich. Den Hosenbund kann er kaum noch schließen. Er arbeitet in einem Versicherungsbüro und hat vor einem Monat einen neuen Chef be-

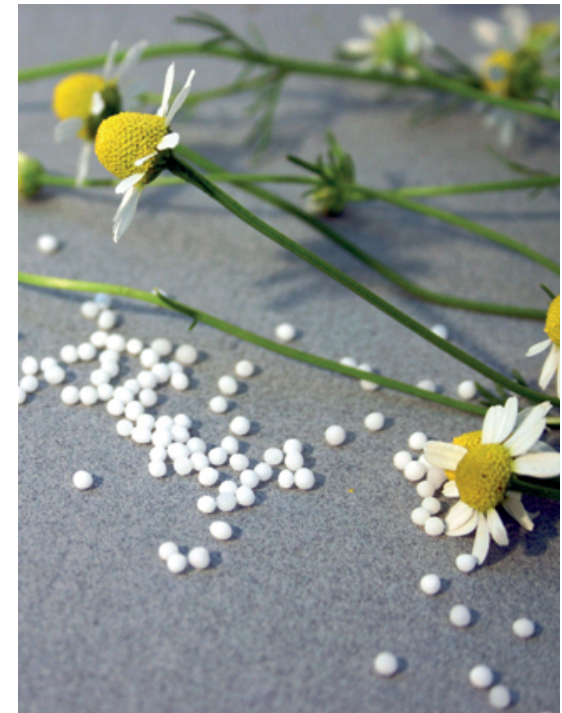

Abb. 2 Bei der Behandlung von Ohren-, Zahnund Bauchschmerzen hat sich Chamomilla bewährt. Foto: (c) Thieme Verlagsgruppe/Michael Zimmermann

kommen. Der macht ihm ziemlichen Druck bez. neuer Versicherungsabschlüsse. Das macht ihn ganz nervös und unruhig.

Gelsemium: Seit einer Woche heftige Kopfschmerzen. Er hatte einen grippalen Infekt, das ist der Rest davon. Er ist sehr erschöpft, kann die Augen kaum offen halten. Nur wenn er Wasser lässt, wird der Kopfschmerz etwas besser, kommt aber dann langsam wieder.

Arnica montana: Seit seinem Unfall vor 5 Wochen Kopfschmerzen. Er hatte eine leichte Gehirnerschütterung mit Übelkeit und Erbrechen. Dann ging es aber nach einer Woche wieder besser. Nur der Kopfschmerz ist geblieben. Wenn seine Frau ihm über den Kopf streichen möchte, geht er 10 Schritte zurück, so unangenehm ist ihm das. Am besten ist es, wenn ihn keiner anfasst.

Belladonna: Seit 2 Tagen heftigste pochende Schmerzen, die von der rechten Schläfe bis zum Ober- und Unterkiefer und in die Zähne ziehen. Sie kommen explosionsartig und sind dann aber irgendwann 
wie weggeblasen. Er ist vor 2 Tagen aus dem Urlaub gekommen. Auf der Fahrt war es sehr heiß, sodass er fast die ganze Fahrt über mit offenem Fenster gefahren ist. Die Hände und Füße sind eiskalt während der Kopfschmerzen. Erschütterung und Bewegung verschlimmern. Ruhe und Liegen im dunklen Zimmer verbessern.

Nux vomica: Seit 1 Woche massive Kopfschmerzen. Alles ist zu viel: Licht, Lärm, Gerüche. Zusätzlich ist er jetzt verstopft.
Wärme und Ruhe bessern. Er hat eine Vortragsreise hinter sich, bei der er 1 Woche lang Vorträge in Florida halten musste. Er hasst das Fliegen, weil ihm die Klimaanlagen nicht bekommen. Er ist gereizt und unbeherrscht.

\section{Gelenkschmerzen - \\ Überlastung, Arthrose etc.}

Bryonia (๑ S. 40-41): Seit ihrem Umzug stechende Schmerzen im linken Knie.
Bewegung, Erschütterung, Kälte verschlechtern. Bewegungslos liegen, feste Binde um das Knie verbessern. Sie ist gereizt, unruhig und hat Durst auf viel kaltes Wasser.

Rhus toxicodendron: Schmerzen in allen Gelenken, seitdem sie den Hausputz gemacht hat. Alles ist steif. Besonders schlimm ist es in der Nacht. Sie muss immer wieder aufstehen und rumlaufen. Nach längerem Sitzen oder morgens nach

\section{Die Mittel im Überblick}

Apis mellifica: Wie nach einem Bienenstich ist alles heiß, rot und entzündlich geschwollen. Bei den Schwellungen handelt es sich immer um Ödeme. Apis hat einen Bezug zur rechten Seite, sodass rechtsseitige Ödeme und Entzündungen besonders angesprochen werden. Berührung und Wärme sind unangenehm, Kälte tut gut. Arnica montana: Patient hat immer das Gefühl wie verprügelt, wie blau geschlagen worden zu sein. Jede Unterlage ist zu hart und schmerzt; deshalb mag der Patient auch keine Berührung, keine Untersuchung. Er hat sogar Angst, nur wenn man auf ihn zugeht. Es gilt als das erste Mittel nach einer Verletzung mit Bluterguss wie Verstauchungen, Prellungen.

Arsenicum album: ist ein ängstlicher Patient, der schnell denkt, dass es jetzt mit ihm zu Ende geht. Er empfindet jede Erkrankung als Bedrohung. Er braucht Betreuung und muss immer über seine Krankheit sprechen. Die Beschwerden beginnen häufig um ca. 1:00 Uhr nachts. Dabei ist der Patient schnell hinfällig, blass, schlapp und sehr kälteempfindlich. Oft betroffen sind der Verdauungstrakt mit Brech-Durchfällen und der Atemtrakt mit asthmatischen Zuständen.

Belladonna: ist ein heftiges Mittel mit plötzlichen, heftigen Beschwerden, die als Schmerzen oder Fieber auftreten können. Sie kommen und gehen plötzlich. Dabei ist der Patient an den Extremitäten kalt, während Kopf und Bauch heiß sind. Die Beschwerden sind i. d. R. auf der rechten Seite lokalisiert. Patienten vertragen keinerlei Bewegung oder Erschütterung. Auch auf der Verhaltensebene reagiert der Patient heftig.
Bryonia: Der Patient liegt still auf der kranken Seite, will keine Störung, keine Bewegung. Fester Druck bessert die Beschwerden. Er hat viel Durst auf kaltes Wasser; ist eher gereizt bei Störungen. Chamomilla: ist ein bewährtes Mittel bei Ohren-, Zahn- und Bauchschmerzen. Dabei ist der Patient extrem schmerzempfindlich und hält gar nichts aus. Das zeigt sich in großer Reizbarkeit aber auch in Ohnmachtsneigung bei Schmerzen.

Colocynthis: fällt auf durch die Modalitäten, dass Druck, Krümmen und Wärme bessern. Er ist ein Mensch, der schnell gekränkt ist und mit Bauchschmerzen darauf reagiert.

Gelsemium: ist das Mittel der Apathie, Muskelschwäche und -zittern in Situationen wie Prüfungen oder grippalen Infekten mit allen Symptomen, die den Infekt begleiten: z. B. Schnupfen, Kopfschmerzen und Fieber. Die Schwäche ist so groß, dass er seinen Augen kaum offen halten kann. Hypericum: Mittel bei Nervenverletzungen durch Sturz, Stoß, Biss oder Stich. Dabei sind die Beschwerden plötzlich, schießen den Nerv entlang. Kälte, Nebel, kalte Luft verschlimmern. Asthma nach Verletzung; Depression nach Verletzung. Ledum: wird gerne als bewährte Indikation bei Tierbissen oder rheumatischen Beschwerden verordnet. Im Gegensatz zu Apis sind die entzündeten Bereiche jedoch kalt, blass und geschwollen. Die Gelenkbeschwerden wandern von unten nach oben. Kälte und kaltes Wasser bessern. Lycopodium: Patient hat sein ganzes Leben lang Verdauungsbeschwerden, was sich bemerkbar macht in
Bauchschmerzen nach blähenden Gemüsen, Ärger, Stress, Kummer etc. Er hat Blähungen und Flatus. Entweder hat er ungeheuren Appetit oder er ist nach einem Bissen satt.

Natrium muriaticum: gilt als ein Kummermittel, wenn der Kummer lange zurückliegt und der Patient darüber gar nicht hinwegkommt. Er ist immer mit Vergangenem beschäftigt, zieht sich zurück und weint allein. $\mathrm{Er}$ kann sich niemandem öffnen. Der Kummer führt entweder zu Kopfschmerzen oder asthmatischen Beschwerden. Dabei verschlechtert die Sonne die Beschwerden, während der Aufenthalt in kühler Luft bessert. Häufig verlangt er salzige Speisen.

Nux vomica: ist das Mittel bei hektischer Lebensweise: viel Arbeit, immer unterwegs, von allem zu viel. Dabei verausgabt sich der Patient, friert schnell und der Körper reagiert mit Beschwerden des Verdauungssystems, des Herzens, der Nerven oder des Atemtrakts. Der Schlaf wird schlecht und der Patient ist cholerisch und übel gelaunt.

Rhus toxicodendron: ist das Gegenteil von Bryonia. Der Patient muss laufen, sich bewegen, sonst „rostet er ein“. Beim Aufstehen nach langem Liegen oder Sitzen ist alles ganz schlimm, durch Bewegung werden die Beschwerden besser. Die Arznei ist bewährt bei Rheuma oder bei allen Erkrankungen, die entstanden sind durch Kälte, nachdem der Patient geschwitzt hat. Ein warmes Bad wirkt entspannend und heilend. 
dem Aufstehen ist es ganz schlimm. Sie muss sich dann erst einlaufen. Am besten ist es in der warmen Badewanne.

Apis mellifica: Nach einem Zeckenbiss ist das rechte Knie ödematös geschwollen, heiß und rot entzündet. Stechende, brennende Schmerzen. Kälte tut gut. Wärme ist ganz schlimm. Sie ist leicht weinerlich, was sie sonst nicht von sich kennt.

Ledum: Rheumatische Beschwerden seit ein paar Wochen: zunächst in den Fußgelenken, dann in den Knien, jetzt in den Handgelenken. Gelenke sind geschwollen, aber blass und kalt. Kühlkissen lindern die Schmerzen; Wärme macht es ganz schlimm. Seitdem sie die Beschwerden hat, ist sie griesgrämig, zieht sich zurück, will keinen Kontakt.

Arnica montana: Seit einem Unfall vor 5 Wochen hat die Patientin heftige Schmerzen im linken Knie. Das Knie fühlt sich verrenkt und wund geschlagen an; jede Unterlage ist zu hart. Sie hat Angst davor, sich

\section{Dosierungsempfehlung}

Je nach Zustand des akut Kranken verordne ich die Mittel in der Potenz C 30 oder C 200. Je heftiger der Schmerz und notwendiger die schnelle Besserung, desto höher wähle ich die Potenz und die Einnahmehäufigkeit. Von dem Mittel werden 3 Globuli in $100 \mathrm{ml}$ Wasser verschüttelt und $1 / 2$ stdl., wenn ganz schlimm $1 / 4$ stdl., schlückchenweise eingenommen bis Besserung eintritt. Die nächste Gabe erfolgt erst wieder, wenn sich der Zustand wieder etwas verschlechtert. Noch nicht so verschreibungssicheren Therapeuten empfehle ich, nicht höher als $C 30$ zu verschreiben.

Je akuter und bedrohlicher ein Zustand ist, desto schneller muss Besserung eintreten. In den hier beschriebenen Fällen sollte die Besserung innerhalb eines Tages eintreten. Nach 4-maliger Einnahme sollte der Patient immer eine beginnende Besserung verspüren. Sonst ist das Mittel nicht richtig. Dann wartet man ca. 1 Stunde ohne Mittelgabe ab und wählt danach ein neues Mittel. untersuchen oder berühren zu lassen. Liegen verbessert den Schmerz.

\section{Rückenschmerzen - Überlas- tung, entzündliche Prozesse etc.}

Belladonna: tobende Schmerzen im Nacken seit Theaterbesuch. Erstrecken sich in die rechte Schulter, ziehen in den rechten Arm. Es war sehr warm und die Klimaanlage war an. Jede Bewegung schmerzt; Ruhe und Wärme tun gut.

Nux vomica: Rückenschmerzen in der Lendengegend, ziehen ins linke Bein hinab. Patient kann sich im Bett nicht umdrehen, muss sich vorher aufsetzen. Kann den Stuhlgang kaum auspressen. Er friert heftig, dabei helfen ihm Wärmekissen. Zudem ist er sehr erregt, wütend und gereizt. Er hat sich fürchterlich über seinen Chef aufgeregt.

Rhus toxicodendron: Nach einem Marathonlauf heftige Rückenschmerzen in der ganzen Wirbelsäule. Es war sehr windig und er war nass geschwitzt. Ruhelos, muss sich immer bewegen, steht ständig auf, kann gar nicht liegen. Bewegung und heißes Baden lindern. Ruhe verschlechtert den Zustand; die erste Bewegung danach ist fast unmöglich.

Bryonia: Brennende Rückenschmerzen im Steißbereich. Der Patient kann sich nicht bewegen. Er muss liegen. Umdrehen im Bett ist unmöglich. Wärme und Ruhe bessern. Er verspürt Durst auf kalte Getränke. Er macht eine Zusatzqualifikation für seinen Beruf und fühlt sich dadurch zunehmend überfordert.
Hypericum: Schneidender, stechend-schießender Schmerz in der Steißbeinregion. Schmerzen schießen entlang der Nervenbahnen ins rechte Bein, der rechte Fuß fühlt sich taub an. Patient kann nicht gehen. Kälte verschlimmert die Beschwerden.

Natrium muriaticum: Seit der Trennung von seiner Frau heftige Rückenschmerzen im Thoraxbereich mit Atemnot. Muss auf einer harten Unterlage liegen, dann werden die Schmerzen besser. Der Patient zieht sich zurück, spricht nicht über die Trennung.

Dieser Artikel ist online zu finden unter: http://dx.doi.org//10.1055/s-0032-1330241

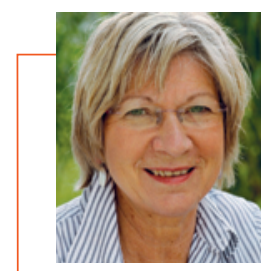

Dr. rer. nat. HP Eva Lang Grüner Grund 1 49536 Lienen

Dr. Eva Lang ist Apothekerin, Heilpraktikerin und in Homöopathie und Psychotherapie ausgebildet. Seit 1985 mit der Homöopathie befasst, seit 1993 in eigener Praxis. Mitinhaberin der Hufelandschule in Senden; Herausgeberin verschiedener Bücher.

E-Mail: e-lang@gmx.de 